\title{
Event Identification in Prototype Fast Breeder Reactor Subsystem using Artificial Neural Network
}

\author{
Subhra Rani Patra \\ Computer Division \\ Indira Gandhi Centre for \\ Atomic Research \\ Kalpakam-603102 \\ Tamilnadu, India \\ T. Jayanthi \\ Computer Division \\ Indira Gandhi Centre for \\ Atomic Research \\ Kalpakam-603102 \\ Tamilnadu, India
}

\author{
R. Jehadeesan \\ Computer Division \\ Indira Gandhi Centre for \\ Atomic Research \\ Kalpakam-603102 \\ Tamilnadu, India
}

\author{
H. Seetha \\ Computer Division \\ Indira Gandhi Centre for \\ Atomic Research \\ Kalpakam-603102 \\ Tamilnadu, India
}

\author{
S. Rajeswari \\ Computer Division \\ Indira Gandhi Centre for \\ Atomic Research \\ Kalpakam-603102 \\ Tamilnadu, India
}

\author{
S.A.V Satya Murty \\ Computer Division \\ Indira Gandhi Centre for \\ Atomic Research \\ Kalpakam-603102 \\ Tamilnadu, India
}

\author{
M. Sai Baba \\ RMG \\ Indira Gandhi Centre for Atomic Research \\ Kalpakam-603102 \\ Tamilnadu, India
}

\begin{abstract}
Identification of events in nuclear power plant is a very challenging task because each event has a unique set of patterns based on the dynamic behavior of the plant. Accordingly the effective set of parameters has to be chosen to identify an event having a particular pattern. This paper describes the development of artificial neural network (ANN) model for event identification of Primary Sodium System of Prototype Fast Breeder Reactor. In reactor under normal operating condition, the Primary Sodium Pump takes the sodium from the cold pool towards the core. Due to some mechanical and electrical problems the Primary Sodium Pump trip and Primary Sodium Pump Seizure may occur which can be identified manually after analyzing the process parameters. The work involves implementation of two ANN models to identify the occurrence of these two events. The effective parameters considered for these two events are SCRAM (Safety Control Rod Accelerated Movement) parameters. The training data for modeling the neural network is prepared using the thermo hydraulics simulation code of PFBR simulator. Multilayer neural network using back propagation algorithm has been widely used for transient identification. The proposed ANN model was able to identify the events correctly and the results obtained are satisfactory.
\end{abstract}

\section{General Terms}

Back Propagation Algorithms, Event Identification

\section{Keywords}

Artificial neural network, Prototype Fast Breeder Reactor, Primary sodium circuit, Back propagation algorithm, Event identification

\section{INTRODUCTION}

Nuclear power plant is a complex and nonlinear system operated by human operators. Using various soft computing techniques the nonlinear dynamic processes of Nuclear Power Plant can be handled. Among those techniques Artificial Neural Network is nonlinear and parametric technique which can model any type of nonlinear system. Only a range of data at different stages of operation is good enough to model a system using Artificial Neural Network. Events are the malfunctions which can affect the safety of nuclear power plant if not identified in time. So when an event occurs the timely and correct detection is of utmost importance in order to avoid the occurrence of any further anomalies and safe status monitoring of the reactor. Our main objective is to develop a neural network model which can identify the events as fast as possible so that the operators can take possible control actions at the earliest $[1,2]$.

A brief study of neural network applications in transient diagnosis is given. Neural network is being used in process fault diagnosis by Sorsa et al [3]. A diagnostic system for identifying accident conditions in a nuclear reactor has been 
developed by T.V Santosh et al [4]. Simulation of Pressurized Heavy Water Reactor data using artificial neural network for reactor status monitoring/ transient identification has been carried out by $\mathrm{P}$ Varde et al. Artificial neural network based system identification and control of nuclear power plant components is carried out by A.G. Parlos et al. [5]. A brief study of neural network applications in transient diagnosis is given by Uhrig et al [6]. Neural network and wavelet transform is being used for fault diagnosis and classification by Kamal H. et al. [7]. Recurrent neural network is trained for identification of anomalous events in a Pressurized Water Reactor 900 Megawatt Nuclear Power Plant (NPP) by Davide Rovero [8]. A dynamic neural network aggregation model is developed for transient detection, classification and prediction in NPP by Kun Mo et al. [9]. Probabilistic ANN is modeled for identification of unlabelled transient in NPP by Mark J. E. et al. [10]. The event identification in a NPP can be detected by two approaches, model based and data driven approach. The model based approach incorporate physical models which detect the fault by checking the consistency between the observed behavior and the predicted behavior through the model $[11,12]$. This work is a part of project, accomplished with the aim of using this application, as a support to operators for safe operation of nuclear reactor systems. It describes development of ANN model for event identification in a 500 MWe Prototype Fast Breeder Reactor Subsystem. The subsystem considered here is Primary Sodium Circuit. The various events which occur in the plant are known as design basis events. Here two design basis events, primary sodium pump trip and primary sodium pump seizure have been considered for detection using ANN models. A range of data has been generated with the help of thermal hydraulics simulation code for the steady state operation and transient operation conditions. The data used as input to train the model has already been validated and recorded in event analysis report of Prototype Fast Breeder Reactor.

\section{NEURAL NETWORK METHODOLOGY}

Artificial neural network is an information processing paradigm consisting of massively interconnected processing elements called neurons working as a whole to solve some specified problems. The processing units are of three types as depicted in figure1: input units which receive signals from outside, output units which sends signals outside the neural network and hidden units which take the data from input units, process the data and then send them to output unit [13]. ANN has a natural propensity for storing knowledge in the connection weights which are connected between the neurons. The knowledge stored in the connections weights is acquired by the training or learning process of the network as time progresses [14].

The neural network is characterized by architecture learning pattern and activation function. The network architecture can be described by the pattern of connection in between the layers and the number of neurons in each layer. The architectures are of two types, feed forward and feedback network. Feed forward network consists of neurons where each layer feeding input to the next layer in forward direction through the connections. Feedback network consists of processing units where the output of each unit is fedback as inputs to all other units forming a loop [15]. The learning process can be explained as the method of adjusting connection weights between the layers of network in order to achieve the outputs of the network. There are three types of learning: Supervised, Unsupervised and Reinforcement learning. In supervised learning the network is provided with a series of inputs and outputs. It is known as learning with teacher. The learning continues till the calculated output matches with the desired output. In unsupervised learning only the inputs are provided without the targeted outputs. It helps in determining the salient features of the network. It is a self organized network without teacher [14]. Reinforcement learning is the mixture of above two. Here we provide the input data which is propagated forward only to tell the network whether the output is right or wrong. If wrong, the weights are adjusted in the midway itself so that later the probability of getting correct output is more in future.

The neural network characteristics include massive parallelism and distributed processing, their robust nature, learning ability, flexibility and adaptability inherent contextual information processing, fault tolerance and low energy consumption etc. The application areas of ANN include pattern completion, classification, feature detection, optimization, approximation, data compression, association, prediction, signal processing, speech production, speech recognition etc.

\section{A BRIEF DESCRIPTION OF PROTOTYPE FAST BREEDER REACTOR}

Prototype Fast Breeder Reactor (PFBR) is a 500 MWe (Megawatt Electrical), Plutonium and Uranium mixed oxide $\left(\mathrm{PuO}_{2}\right.$ and $\left.\mathrm{UO}_{2}\right)$ fuel, sodium cooled, pool type reactor. As shown in figure 2, PFBR flow sheet describes the various subsystems associated with it and their interconnections [16]. The heat transport system of PFBR consists of primary sodium circuit, secondary sodium circuit and steam water system. The primary sodium circuit is contained inside the main vessel of the reactor. It consists of two primary sodium pumps and four Intermediate Heat Exchangers (IHX). The main objectives of primary sodium main circuit are:

- To transfer the nuclear heat generated in the core to the secondary sodium circuit through IHX keeping in mind the maintenance of safe operating temperature of core subassemblies and main vessel.

- To ensure safe operating temperature of the core subassembly and main vessel by transferring the decay heat from the core subassembly to secondary sodium circuit.

Figure 1 shows the PFBR flow diagram having all the components of the plant. 


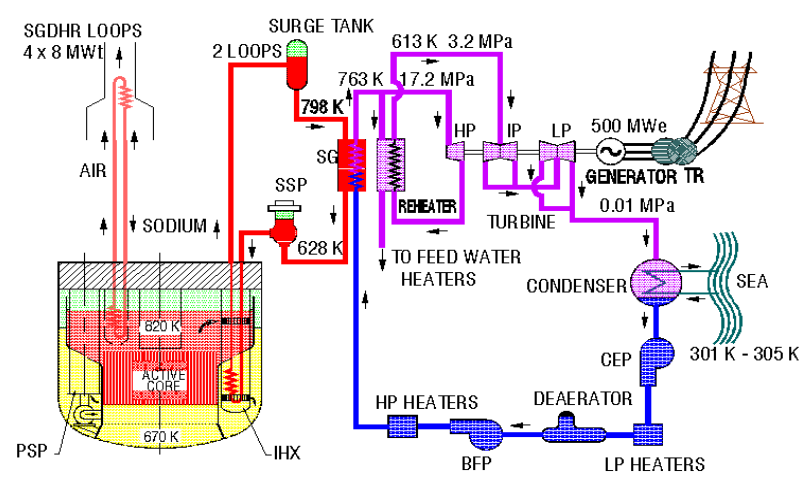

Fig1. PFBR flow sheet

The secondary sodium system consists of surge tank, four steam generators (SG) and secondary sodium pump. The steam water system consists of turbine with separate reheaters which employ regenerative feed water heating.

\subsection{Description of Primary Sodium Pump}

The Primary sodium pump is a single top suction, single stage vertical shaft centrifugal pump placed in cold sodium pool [17]. Each pump is located between two IHX around the core. The pumps operate in parallel across the core. The nominal speed of the pump is $590 \mathrm{rpm}$. The operating temperature of the pump is $670 \mathrm{k}$ and the fluid inside the pump is liquid sodium. The primary sodium pump and its operation are shown in figure 3. In figure 2 the schematic of Primary Sodium Pump is shown.
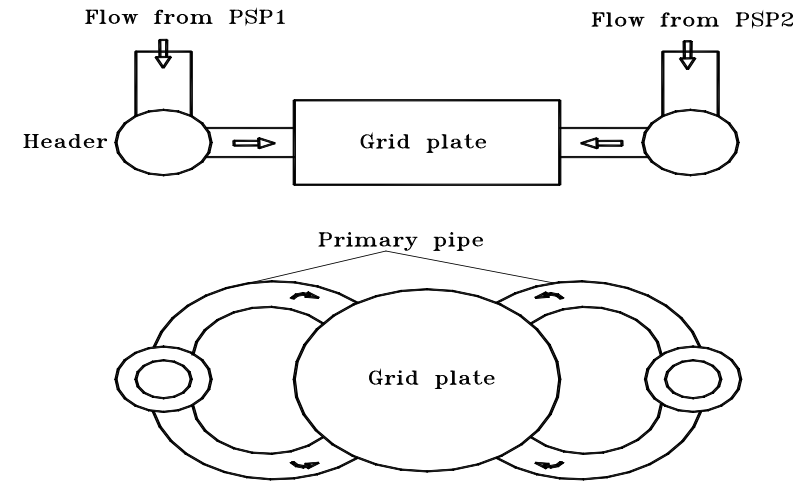

Fig2. Schematic of Primary Sodium Pump

\subsection{Events associated with Primary Sodium Pump}

The safety of PFBR is evaluated by the response of plant to various events which affect the plant and they are known as Design Basic Events (DBE). The DBEs are classified into 4 categories namely category 1 (all planned conditions), category $2\left(>10^{-2} /\right.$ reacor year (ry)), Category $3\left(\leq 10^{-2} /\right.$ ry but $>10^{-4} /$ ry $)$ and Category 4 events $\left(\leq 10^{-4} /\right.$ ry but $>10^{-6} /$ ry $)$. There are various events associated with Primary Sodium Pump. Two important events among those, PSP trip and PSP seizure have been considered for modeling. Figure 4 shows the event analysis graphs of PSP trip and PSP seizure event. PSP trip is a category 2 event. The probability of occurrence of the event is high compared to category 3 and 4 . It may happen due to mechanical problems such as misalignment in flywheel, bearings and seismic events etc. and electrical problems like fault in power supply and failure in pony motor etc. PSP seizure is a category 3 event. This may occur by some mechanical faults like insufficient hydrostatic bearing clearance, seismic events, foreign particle etc [18].

\subsubsection{One Primary Sodium Pump (PSP) Trip}

When one PSP trip occurs, the speed of the tripped PSP flow reduces gradually against inertia to $50 \%$ in $2.6 \mathrm{~s}$ and to $0 \%$ in 9.4s. Due to parallel operation of two PSPs the operating PSP flow increases to $126 \%$ in order to balance the core flow. The total core flow reduces to $61 \%$ in 10 s. Hence the power to flow ratio $(\mathrm{P} / \mathrm{Q})$ increases and then the central subassembly outlet temperature $\left(\theta_{\mathrm{CSA}}\right)$ increases which leads to increase in central subassembly temperature rise $\left(\Delta \theta_{\mathrm{CSA}}\right)$ and mean core temperature rise $\left(\Delta \theta_{\mathrm{M}}\right)$. The hot pool sodium temperature increases, which in turn increases the cold pool sodium temperature or reactor inlet temperature finally leading to safety controlled rod accelerated movement (SCRAM). Out of a set of SCRAM parameters five effective SCRAM parameters viz. Np (Pump speed), $\mathrm{P} / \mathrm{Q}, \theta_{\mathrm{CSA}}, \Delta \theta$ CSA and $\Delta \theta_{\mathrm{M}}$ are used for protection of this event. Among the various parameters present the first appearing parameters that independently trigger reactor SCRAM by shutdown systems are $\mathrm{Np}$ and $\theta_{\mathrm{CSAM}}$ respectively. The reactor is designed to ensure that the design safety limits are not exceeded during the normal operational range.

\subsubsection{One Primary Sodium Pump (PSP) Seizure}

When a PSP Seizure occurs there is a ramp reduction of the speed of one pump to zero in one second. The second pump is considered to be continuing to operate at full speed. The operating PSP flow increases to $125 \%$ and the core flow reduces to $37 \%$ in $1.7 \mathrm{~s}$. With decrease in flow in such a fast rate the sodium temperature increases very rapidly. Out of several number of SCRAM parameters six effective parameters viz. $\mathrm{N}_{\mathrm{P}}, \mathrm{P} / \mathrm{Q}, \theta_{\mathrm{CSAM}}, \rho$ (reactivity), $\Delta \theta_{\mathrm{CSAM}}$, and $\theta_{\mathrm{M}}$ parameters are available during the event. A speed sensor is mounted at the pump shaft so as to continuously measure the speed during the event and hence $\mathrm{Np}$ is the most significant SCRAM parameter of Shutdown system 1 . And $\theta_{\mathrm{CSAM}}$ is most significant parameter of Shutdown system 2 because the temperature sensing time of the thermocouple of central subassembly is very less.

\subsubsection{Analysis of Design Basis Events}

The figures below show various SCRAM parameters and the time at which they cross the threshold values. It can be observed from the graph that for PSP trip the Np parameter crosses its threshold of $95 \%$ at $0.45 \mathrm{~s}$ and for PSP seizure it crosses the threshold at $0.05 \mathrm{~s}$. Similarly the P/Q parameter crosses its threshold of 1.1 at $1.6 \mathrm{~s}$ for PSP trip and at $0.21 \mathrm{~s}$ for PSP seizure. Threshold values for SCRAM parameters are indicated in the graph. Figure 3 depicts the PSP trip and PSP seizure event related graphs. 


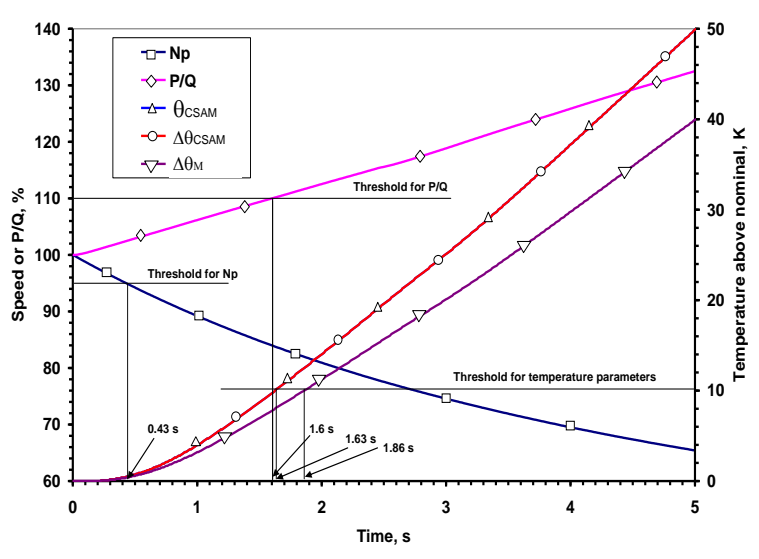

(a)

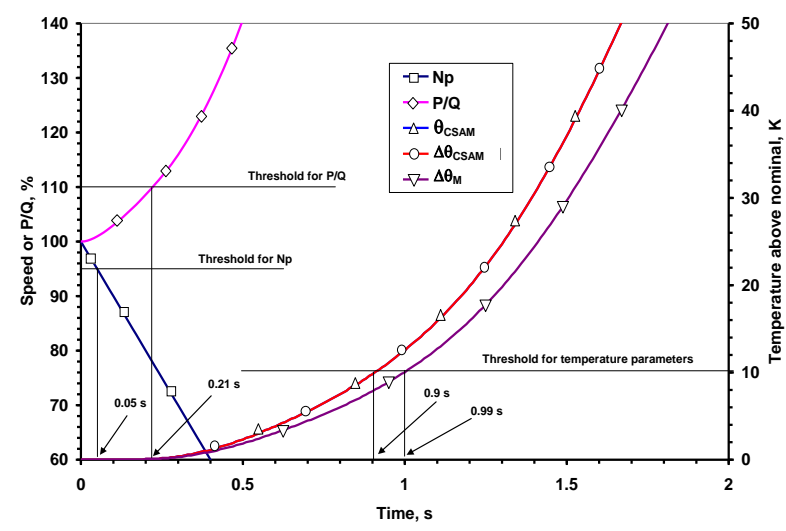

(b)

Fig 3: Evolutions of process values of SCRAM parameters during one (a) PSP trip and (b) PSP seizure event

\section{TRAINING and FINE TUNING OF THE NEURAL NETWORK}

Multilayer feed forward neural network architecture using back propagation algorithm has been used for event identification of primary sodium circuit. In back propagation algorithm the mathematical formula presented can be applied to any network and does not require any special mention of the features of the function to be learnt. The batch update weights in back propagation provides a smoothing effect on the weight correction terms.A network is said to be generalized when it sensibly interpolates with input connections that are new to the network. The best network for generalization is back propagation network $[14,19]$. Figure 4 shows multilayer neural network diagram.

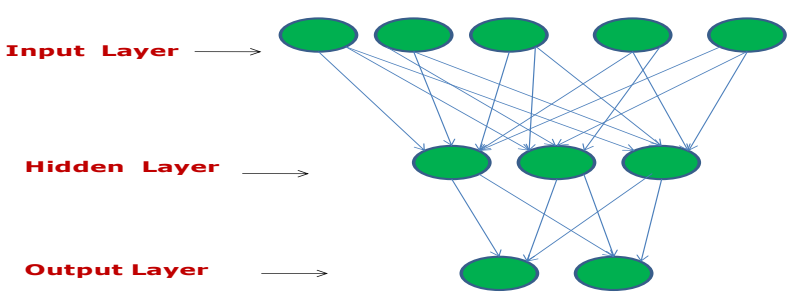

Fig4. Multilayer Neural Network
All the relevant parameters causing the occurrence of the events have been taken as inputs to the input layer of neural network. The output of the neural network is that particular event which has to be identified. The network has been trained such that when the event occurs the output is zero. For PSP trip event, the neural network is having five input nodes and one output node. The input parameters are Np, P/Q, $\theta_{\text {CSA }}$ and $\Delta \theta_{\mathrm{CSA}}, \Delta \theta_{\mathrm{M}}$. Likewise the inputs for PSP seizure event are six, Np, P/Q, $\theta_{\mathrm{CSA}}, \Delta \theta_{\mathrm{CSA}}, \Delta \theta_{\mathrm{M}}$ and $\rho$. The transient data is generated using thermo-hydraulics code. It is then divided into training samples and testing samples in (80\%:20\%) ratio approximately. Since the data cover a wide range, it has to be normalized between zero to one. The normalization formula used here is given in equation 1 .

$$
D_{\text {norm }}=\frac{D-D_{\min }}{D_{\max -D \min }}
$$

After normalization the data is fed to the neural network and random weights are assigned first. The input signals move from input layer to output layer through hidden layer. The inputs after getting multiplied with the random weights are summed up. The summed up value is then fed to the non linear activation function which is a sigmoid function here. It is represented by equation 2 .

$$
\frac{2}{1+\exp (-x)}-1
$$

Where $x$ is the summed up result of the weight multiplied with inputs.

The result is then compared with the desired output and if it disagrees with the desired output the difference in desired and actual output is back propagated and put in the weight change step. The weight is then updated by adding the change in weight to the previous weight. Learning rate parameter is applied to the weight change factor in order to get faster convergence. Following equations 3 (a) and 3 (b) give the weight updated value.

$$
\begin{aligned}
& W_{k j}=W_{k j}+\delta W_{1} \\
& W_{j i}=W_{j i}+\delta W_{2}
\end{aligned}
$$

Where $W_{k j}$, is the weight applied from hidden to the output layer and $W_{j i}$ is the weight change from input to hidden layer and $\delta W_{1}, \delta W_{2}$ are the respective change in the weight vector. The training process is repeated till the stopping criteria are being met, when the mean square error (MSE), the difference in desired and calculated output becomes negligibly small. The MSE can be represented as equation 4 .

$$
M S E=\frac{1}{T S N} * \sum_{t=1}^{T S N} \sum_{k=1}^{N O N}\left(\left(d_{o u t}\right)_{k t}-O_{k t}\right)^{2}
$$

Where $T S N$ represents the number of training samples, $N O N$ represents the number of output nodes, dout and $O$ represents desired and actual outputs. 
In case of PSP trip 49 samples are used for training. The neural network developed for PSP trip event has three layers. Input layer consists of 5 input nodes, hidden layer can be varied and fine tuned and output layer consists of one output, i.e., the event itself. In case of PSP seizure 54 samples are used for training the neural network. The input layer consists of 6 input nodes and output layer is of one output node, the event. Both the training and testing samples cover the range of data from steady state to transients. The network is trained using the back propagation algorithm and the coding has been done in C-programming.

The fine tuning has been carried out by various trials for finding the optimal number of hidden nodes and learning rates leading to least mean square error. For the ANN model which detected the PSP trip event the various learning rates used for trial ranges from 0.001 to 0.1 and the optimal learning rate is found to be 0.05 . Similarly the number for hidden nodes have been varied from 4 to 12 by keeping the learning rate constant and the results are analyzed to find out the optimal number of hidden nodes. With 11 hidden nodes in the hidden layer the network converges faster for the given configuration. Likewise for the ANN model developed to detect PSP seizure event the learning rates are varied from 0.001 to 0.2 and hidden nodes are varied from 5 to 13 and the optimum values are achieved at number of hidden nodes $=12$ and learning rates $=0.005$. Choosing number of hidden nodes in hidden layer is of major concern. In this case, many networks have been tried with different number of hidden nodes, and generalization error has been estimated for each network. The network having optimal number of hidden nodes and least generalization error has been chosen as fine-tuned network.

\section{RESULTS AND DISCUSSION}

The identification of PSP trip and PSP seizure events have been considered for modeling and two separate ANN models have been implemented. In PSP trip event five system parameters have been used as inputs and in PSP seizure event six system parameters have been used including the reactivity parameter which is of major concern for the SCRAM to occur. The weight parameters achieved from the training process are used in the testing phase with a set of test samples which has not been trained earlier.

\subsection{Case 1: PSP TRIP}

The trial results are shown in figure 5. The optimal mean square error value of $2.83 \mathrm{e}-05$ is achieved with number of hidden nodes $=11$ and learning rate $=0.05$ for 10 lakh iterations. The training time for 20 lakh iterations was 10 minutes in a $2.66 \mathrm{Ghz}$ Intel Core 2 Duo PC. The figure 6 shows that the actual outputs obtained during training are almost matching with the desired outputs after 20 lakh iterations.

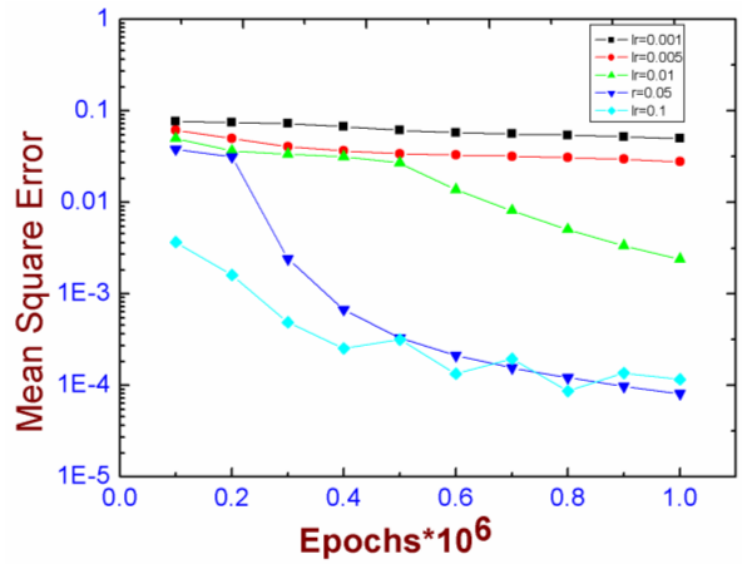

(a)

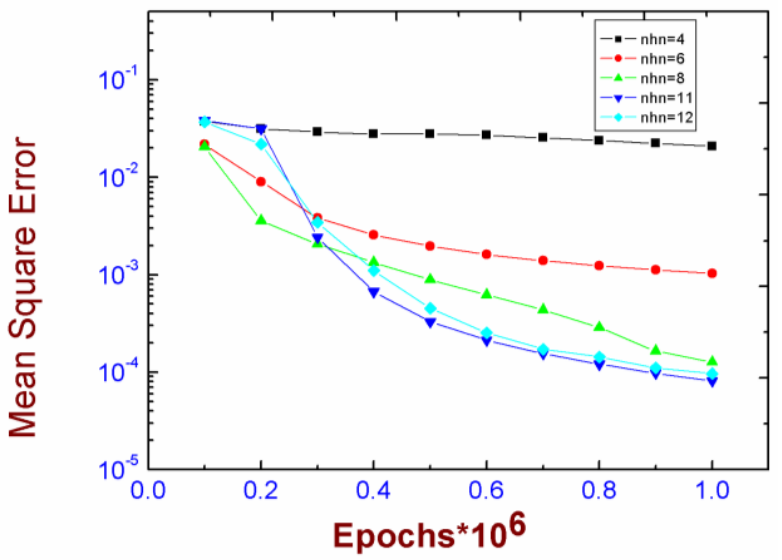

(b)

Fig5. Graph plotted between mean square error and number of epochs for (a) various learning rates (b) number of hidden nodes 


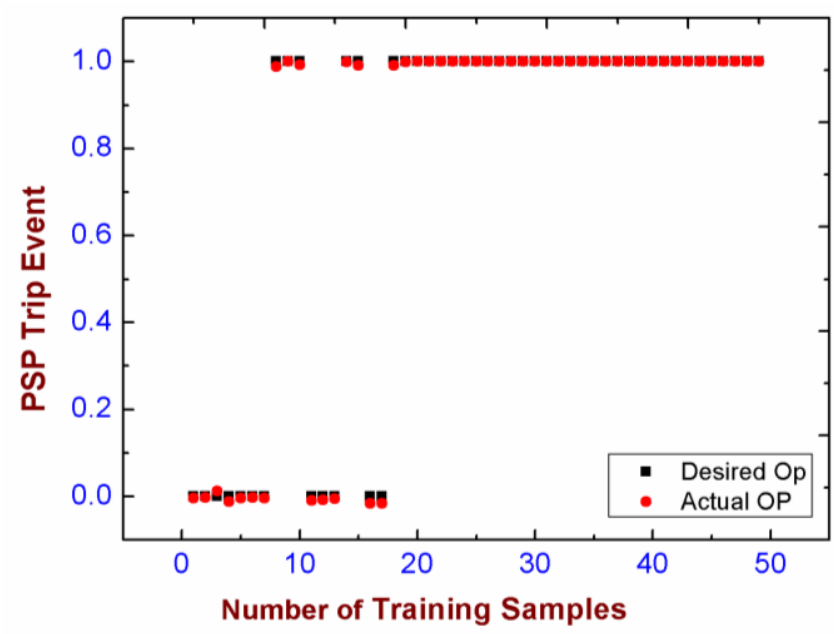

Fig6. Neural Network training results for PSP trip event showing desired and actual output

Four distinct test samples from the trained input range are used for testing the network. The neural network model is then validated against those test samples and the results show that the occurrences of PSP trip can be identified with negligible error as shown in figure 7. In the graphs, occurrence of the event is identified by output 1 and the steady-state condition with output 0 .

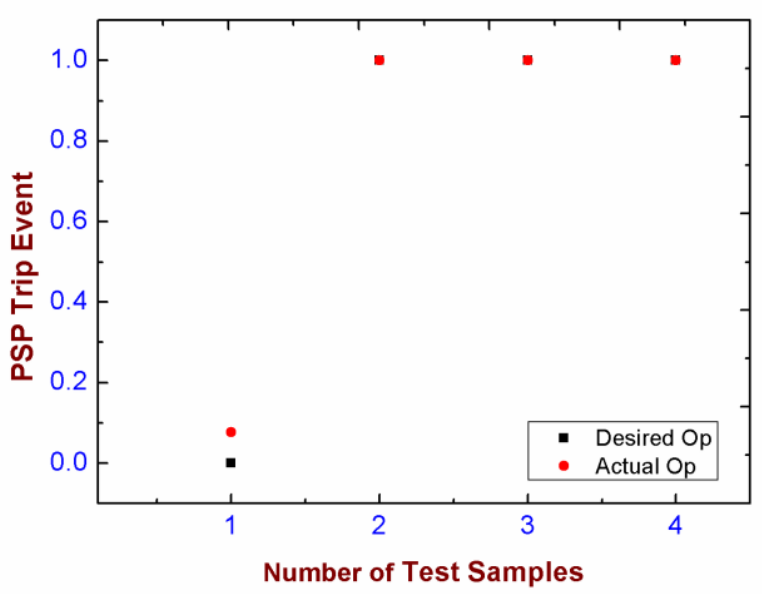

(a)

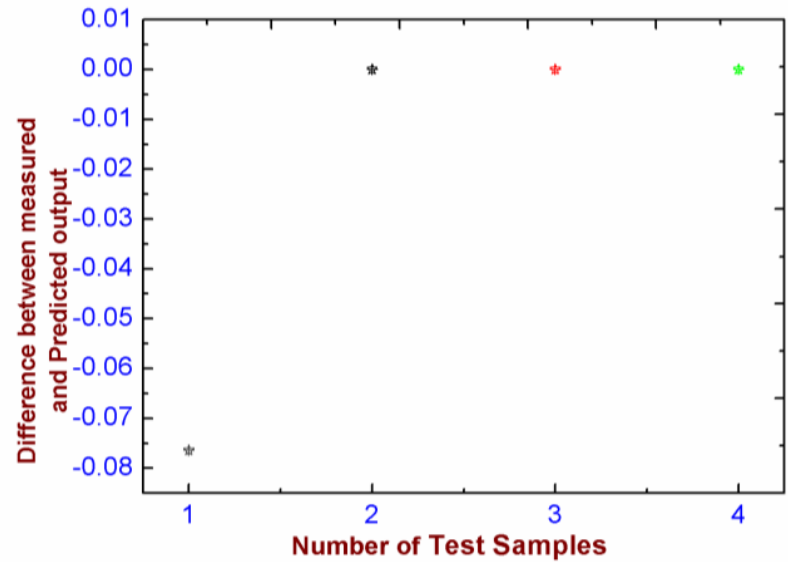

(b)

Fig7. Test results for PSP Trip event neural network model showing (a) desired and actual output and (b) the difference between desired and actual output 


\subsection{Case 2: PSP SEIZURE}

The trial results are shown in figure 8 . The optimal mean square error value of $2.83 \mathrm{e}-05$ is achieved with number of hidden nodes $=12$ and learning rate $=0.005$ for 10 lakh iterations. The figure 9 depicts the training results of PSP seizure event. From the graph it is shown that the desired and actual outputs are in well agreement with each other.

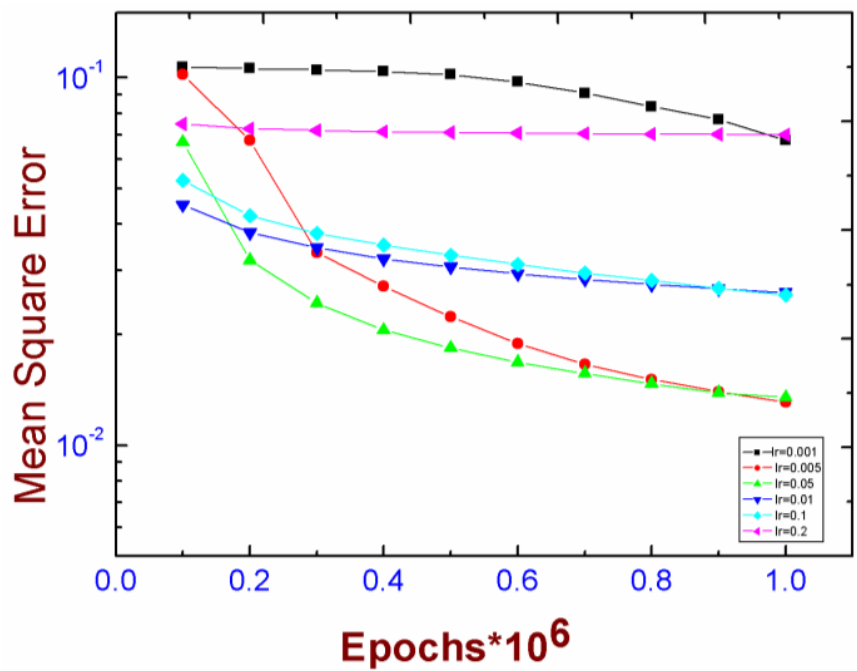

(a)

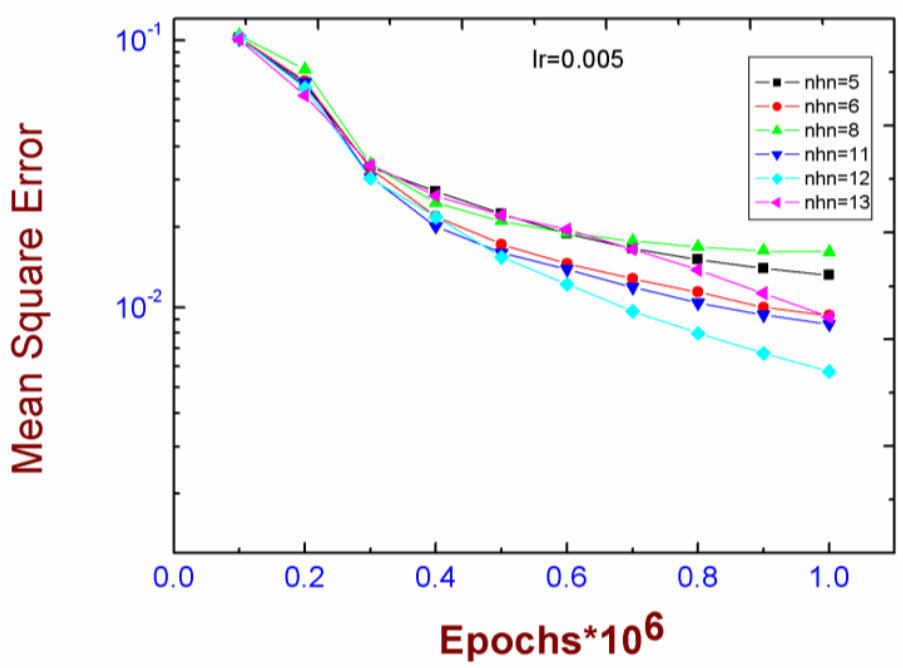

(b)

Fig8. Graph plotted between mean square error and number of epochs for (a) various learning rates (b) number of hidden nodes 


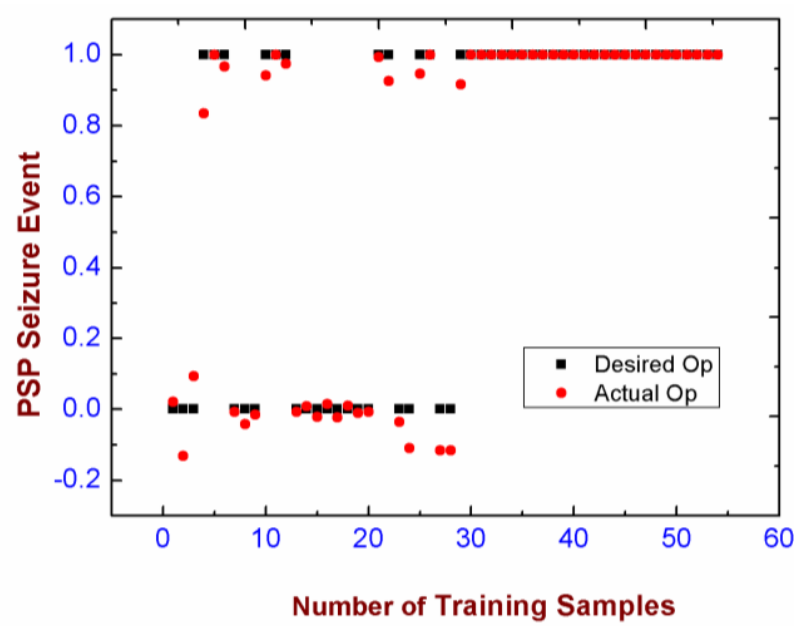

Fig9. Neural Network training results for PSP Seizure event showing desired and actual output

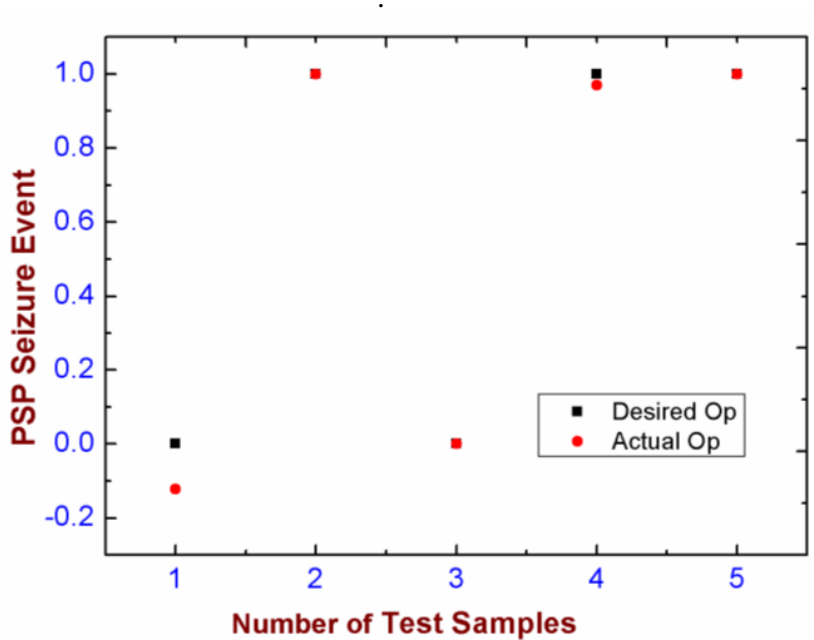

(a)

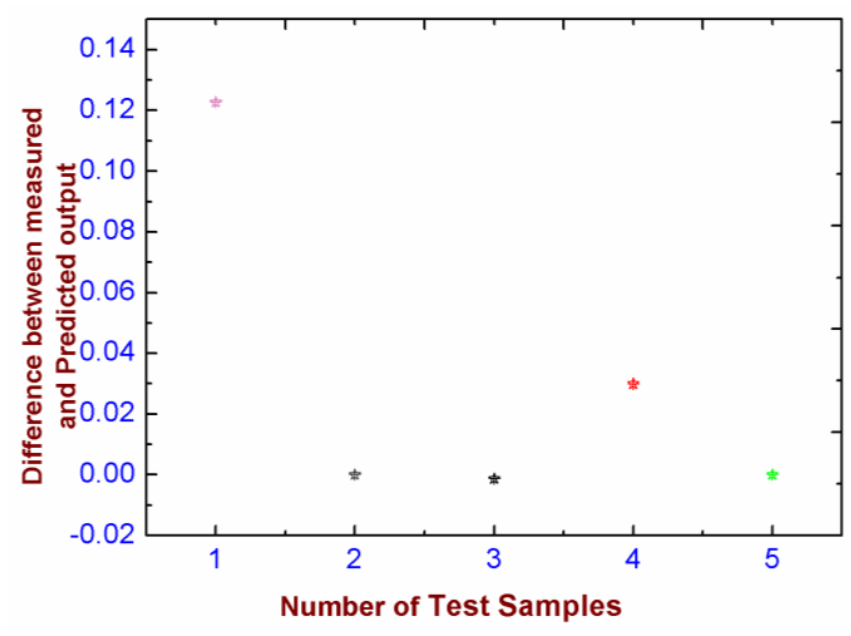

Fig10. Test results for PSP Seizure event neural network model showing (a) desired and actual output and (b) the difference between desired and actual output
Five distinct test samples from the trained range are used for validation. The test results shown in Fig. 10 indicate that the desired output almost matches with the actual output and the occurrence of the events can be identified satisfactorily. Multilayer feed forward ANN model has been implemented and trained with BP algorithm to identify events related to PFBR subsystem. From the results it is shown that the neural network gives comparatively faster results than obtained from the point kinetics equation model.

\section{CONCLUSION}

This paper describes the identification of events for Primary Sodium Circuit in PFBR using Artificial Neural Network model. The steady state and transient data have been collected from the thermo hydraulics model of PFBR simulator. The effective SCRAM parameters which cause the events are used as inputs to the neural network. Two separate ANN models have been developed to detect PSP trip and PSP seizure events. Multilayer feed forward network using back propagation algorithm is implemented and training has been carried out to achieve least mean square error with optimal network parameters. The occurrence of events has been successfully detected during the validation of our ANN model. The network once trained properly, is ready to identify the transient in much less time compared to identification of events detected manually. Hence these models can be very helpful in quick detection and corrective decision making during the operation of nuclear reactors. Our future aim is to identify the all associated events by integrating individual subsystem models into a single neural network model.

\section{ACKNOWLEDGMENTS}

We are immensely thankful to Dr. P. Swaminathan, Director, Electronics \& Instrumentation Group and Dr. Baldev Raj, Director, Indira Gandhi Center for Atomic Research, (Department of Atomic Energy), Kalpakkam for their constant support and guidance for this project. 


\section{REFERENCES}

[1] Santosh T.V., Srivastava A., Sanyasi Rao V.V.S., Ghosh A.K., Kushwaha H.S.,2009, Diagnostic system for identification of accident scenarios in nuclear power plants using artificial neural networks, Reliability Engineering and system Safety, 759-762.

[2] Varde P.V, Chowdhury R., Vinod G, Babar A.K, Kushwaha H.S, Simulation of Pressurized Heavy Water Reactor Data Using Artificial Neural Network for Reactor Status/Transient Identification, International Conference on Quality, Reliability and Control(ICQRC2001),http://www.barc.ernet.in/publications/n1/2002/200 210-08.pdf

[3] Sorsa T., Koivo H.N., Koivisto H., Neural Networks in process Fault Diagnosis, 21,1991, IEEE Transactions on Systems, Man and Cybernetics,815-824,2002, http://ieeexplore.ieee.org/xpl/freeabs_all.jsp?arnumber=1 08299\&abstractAccess $=$ no\&userType $=$ inst.

[4] Santosh T.V., Kumar M., Thangamani I., Srivastava A., Dutta A., Verma V., Mukhopadhyay D., Ganju S., Chatterjee B., Rao V.V.S.S., Lele H.G., Ghosh A.K., A diagnostics system for identifying accident conditions in a nuclear reactor, Nuclear engineering and design, 241, 177-184, 2011

[5] Parlos A.G., Fernandez B., Artificial neural networks based system identification and control of nuclear power plant components, Proceedings of IEEE $29^{\text {th }}$ conference on decision and control, 1703-1706, Vol. 3. http://ieeexplore.ieee.org/Xplore/defdeny.jsp?url=http\%3 A\%2F\%2Fieeexplore.ieee.org\%2Fstamp\%2Fstamp.jsp\% 3Ftp\%3D\%26arnumber\%3D203911\%26userType\%3D\& denyReason $=-$

133\&arnumber=203911\&productsMatched=null\&userTy $\mathrm{pe}=$

[6] Attieh I.K., Gribok A.V., Hines J.W., Uhrig R.E., Pattern recognition techniques for transient detection to enhance nuclear reactor's operational safety, Nuclear engineering and design, 207, 207-221, 2001.

[7] Hadad K., Pourahmadi M., Majidi-Marahgi H., Fault diagnosis and classification based wavelet transform and neural network, Progress in nuclear energy, 53, 41-47, 2011

[8] Roverso D., Neural ensembles for event identification, Proceedings of safeprocess, IFAC symposium on fault detection, supervision for technical process, 2000
[9] Mo K., Leo S. J., Seong P.H., A dynamic neural network aggregation model for transient diagnosis in nuclear power plants, Progress in nuclear technology, 49, 262272,2007

[10] Embrechts M.J., Benedek S., Hybrid identification of unlabelled nuclear power plant transients with artificial neural networks, Neural network proceedings, The 1998 IEEE International Joint Conference on IEEE World Congress on Computational Intelligence, 2, 1438 - 1443

[11] Zio E., Maio F D., Stasi M., A data-driven approach for predicting failure scenarios in nuclear systems, Annals of nuclear technology, 37, 482-491, 2010.

[12] Ma J., Jiang J., Applications of fault detection and diagnosis methods in nuclear power plants: A review, Progress in nuclear energy, 53, 255-266, 2011

[13] Krose B., Patrick V.D.S., An introduction to neural network, page-15, 1996, http://www.cs.unibo.it/babaoglu/courses/cas/resources/tu torials/Neural_Nets.pdf

[14] Sivanandam S.N., Sumathi S., Deepa S.N., Introduction to neural networks using MATLAB 6.0, 20-21, 2009, Tata McGraw Hill Edu. Pvt. Ltd

[15] Yegnanarayana B, Artificial Neural Networks, 88-142, 2008, Prentice-Hall India pvt. Ltd.

[16]Raghupathy S., Singh O.P., Govindarajan S., Chetal S.C., Bhoje, Design of 500 MWe Prototype Fast Breeder Reactor, http://www.dae.gov.in/ni/nimar04/design.pdf, 2004

[17] Chetal S.C., Bhoje S.B. etal, Conceptual design of Heat Transport Systems and components of PFBR-NSSS, http://www.iaea.or.at/inisnkm/nkm/aws/fnss/fulltext/280 14320.pdf, 1995

[18]N Kasinathan, U Parthasarathy., K Natesan., P Selvaraj, P Chellapandi, S C Chetal and S B Bhoje, Internal Report on "Analysis of Design Basis Events for Prototype Fast Breeder Reactor", $1^{\text {st }}$ National Conference on Nuclear Reactor Safety, Mumbai, Nov 2002.

[19] Sivanandam S.N., Deepa S.N., Principles of Soft Computing, 74-82, 2010, Tata McGraw Hill Edu. Pvt. Ltd 